\title{
OPEN Infection effects of the new microsporidian species Tubulinosema suzukii on its host Drosophila suzukii
}

\begin{abstract}
Sarah Biganski ${ }^{1}$, Sabrina Fückel ${ }^{1,2}$, Johannes A. Jehle ${ }^{1}$ \& Regina G. Kleespies ${ }^{1 \bowtie}$
Microsporidian infections of insects are important natural constraints of population growth, often reducing lifespan, fecundity and fertility of the infected host. The recently discovered Tubulinosema suzukii infects Drosophila suzukii (spotted wing drosophila, SWD), an invasive pest of many fruit crops in North America and Europe. In laboratory tests, fitness effects on larval and adult stages were explored. High level infection after larval treatment caused up to $70 \%$ pupal mortality, a decreased lifespan and a $70 \%$ reduced oviposition of emerging adults in biparental infection clusters. A shift to higher proportion of female offspring compared to controls suggested a potential parthenogenetic effect after microsporidian infection. A clear sex-linkage of effects was noted; females were specifically impaired, as concluded from fecundity tests with only infected female parents. Additive effects were noted when both parental sexes were infected, whereas least effects were found with only infected male parents, though survival of males was most negatively affected if they were fed with $T$. suzukii spores in the adult stage. Although most negative effects on fitness parameters were revealed after larval treatment, infection of offspring was never higher than $4 \%$, suggesting limited vertical transmission. For that reason, a self-reliant spread in natural SWD populations would probably only occur by spore release from cadavers or frass.
\end{abstract}

Microsporidia are spore-forming, obligate intracellular parasites early evolved presumably as a basal linage or a sister taxon to Fungi ${ }^{1-4}$. They infect a huge range of arthropod species but also vertebrates including humans ${ }^{5}$. Oral ingestion of spores is the major form of horizontal transmission where spores enter the host cell cytoplasm by penetration of midgut cells after everting the polar tube. Other infection routes via vertical transmission are either transovarial: inside egg or embryo ${ }^{6}$, transovum: on the surface of the egg chorion ${ }^{7,8}$, or venereal transmission: paternal ${ }^{9}$. To reproduce and spread within the host tissue, microsporidia undergo several morphological changes from meronts (proliferation) to spores (sporogony) ${ }^{8,10}$. Severe infections located in one or more host tissues or organs can lead to highly negative health effects on the host, as it is known for Nosema disease in honeybees causing heavy intestine disorders, shortened lifespan and neuronal/behavioural changes leading to disorientation ${ }^{11-15}$. Though, microsporidia are rarely fast-killing pathogens, they are able to reduce host populations by weakening the host fitness such as reduction of fertility and offspring, growth rate, and life span. Horizontally transmitted microsporidia frequently show higher virulence than vertically transmitted, and is commonly favoured by high host population density, e.g. honeybees and gypsy moth populations ${ }^{16,17}$. Vertical transmission is frequently found with microsporidia with low virulence, depending on host survival, fertility and number of infected offspring to ensure their own reproduction ${ }^{18,19}$. Vertical transmission has been noted for at least fourteen Microsporidia taxa $\mathrm{a}^{20}$ and is known to reduce fecundity and fertility in several insect hosts ${ }^{21-24}$. Although there are some microsporidian species that increase mortality of their insect host, their usage as pest control agent in plant protection has been rarely considered. Paranosema (Nosema) locustae, infecting acridids, is an example of efficient but also problematic use of microsporidia in biological control (reviewed by Lockwood et al. $^{25}$ ).

Due to human activity, travel and worldwide transport of agricultural products, but also climate change, the spread of pest insects of cultivated plants has been highly facilitated, resulting in the invasion of new pests and diseases to previously unaffected regions ${ }^{26}$. In about a decade the spotted wing Drosophila (SWD), Drosophila

\footnotetext{
${ }^{1}$ Federal Research Centre for Cultivated Plants, Institute for Biological Control, Julius Kühn Institute, Heinrichstraße 243, 64287 Darmstadt, Germany. ${ }^{2}$ Technische Universität Darmstadt, Schnittspahnstraße 10, 64287 Darmstadt, Germany. ${ }^{\square}$ email: regina.kleespies@julius-kuehn.de
} 


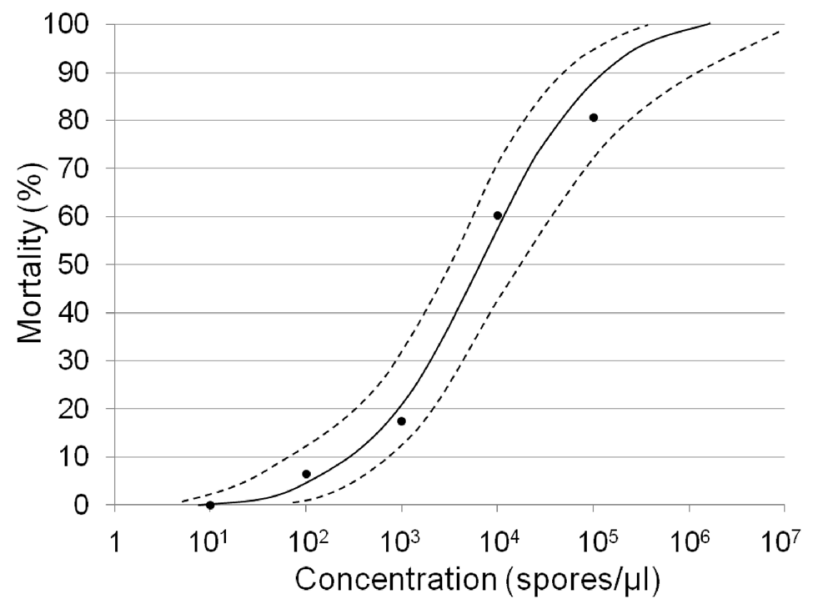

Figure 1. Spore concentration-mortality response of D. suzukii second instar larvae, 18 days after exposure to T. suzukii spores. Independent replicates/number of tested individuals $(\mathrm{R} / \mathrm{N})$ : untreated control: $\mathrm{R} / \mathrm{N}=9 / 252$, $10^{1}$ spores: $\mathrm{R} / \mathrm{N}=2 / 96,10^{2}$ spores: $\mathrm{R} / \mathrm{N}=5 / 120,10^{3}$ spores: $\mathrm{R} / \mathrm{N}=6 / 180,10^{4}$ spores: $\mathrm{R} / \mathrm{N}=7 / 211,10^{5}$ spores: $\mathrm{R} / \mathrm{N}=2 / 24)$. Logarithm $\log (\mathrm{x})$ of concentrations and number of organisms entered the probit analysis using linear maximum likelihood regression. Slope function was calculated after Litchfield and Wilcoxon. Concentration-response: $F=76.966, D F=3, P(F)=0.003$. Dots = observed mortality, solid line = calculated concentration, dashed lines = upper and lower $95 \%$ confidence limits.

suzukii ${ }^{27}$, has become a major pest in commercial soft-skinned fruit orchards in Europe, North and South America and $\mathrm{Asia}^{27-29}$. Naturally occurring antagonists, both metazoans ${ }^{30,31}$ and microorganisms, may play an important role for biological control of SWD but efficient microbial antagonists have not been reported ${ }^{31-33}$. Antagonistic fungi and bacteria have been screened but also criticized for inefficiency and/or inappropriate application ${ }^{33-35}$. In addition, some viruses have been identified from SWD that should be examined for their potential as biocontrol agents ${ }^{36,37}$.

Recently, a microsporidian infection was discovered in SWD flies originating from Oregon, $\mathrm{USA}^{38}$ and a new species Tubulinosema suzukii (family: Tubulinosematidae) was described ${ }^{39}$. Here, we report laboratory experiments to explore the potential effect of T. suzukii infection on fitness parameters of SWD, including eclosion and survival rates, egg production and offspring rates, when infections were initiated in larval and adult stages. We found that every fitness parameter tested was strongly impacted when infection began in the larval stage.

\section{Results}

Median lethal concentration ( $L C_{50}$ ). Inoculation of second instar SWD larvae (L2) with suspensions of five different spore concentrations of T. suzukii resulted in a $\mathrm{LC}_{50}$ of approximately $6.9 \times 10^{3}$ spores/ $\mu \mathrm{l}(95 \%$ confidence limits $(\mathrm{CL})=3.4 \times 10^{3}-1.7 \times 10^{4}$ spores $/ \mu \mathrm{l}, \mathrm{N}=631$, slope $=0.915, \chi^{2}=21.683$ ) (Fig. 1 ). Mortality rates were determined by failure of the emergence of imagines because death of larvae could not be determined within the growth medium. Control mortality was $28.2 \%$ after 18 days post inoculation (dpi). Maximum mortality was $80 \%$ at the highest spore concentration applied.

Quantification of infection process. To study the infection process, SWD L2 larvae were inoculated with $4 \times 10^{4}$ spores, followed by DNA extraction from larvae, pupae and adults for quantitative real-time PCR (qPCR) (Fig. 2a) and examination by microscopy (Fig. 2b). In third instars (L3) at 3-5 dpi, DNA copies equivalent to $1.33 \pm 1.22 \times 10^{6}($ mean $\pm S E$ ) extracted spores were recorded (Fig. 2a). In early pupal stage microsporidian DNA increased to $8.54 \pm 7.12 \times 10^{6}$ copies and was stable until late pupal stage (8-13 dpi) with $6.8 \pm 2.97 \times 10^{6}$ copies per SWD pupa. After the transition between late pupal stage to adulthood (13-20 dpi), the DNA amount increased by the factor of 20 in early adult stage with $13.3 \pm 7.91 \times 10^{7}$ copies per individual and was significantly increased compared to DNA copies at 3-5 dpi (GLM analysis for gamma distribution with log-link function: $\chi^{2}=14.014, D F=3, P=0.003$, Tukey test: $3-5$ dpi-13-20 dpi: $\left.P<0.001\right)$. Correspondingly, Giemsa-stained tissues of early larvae ( $6 \mathrm{dpi}$ ) revealed just a few free developmental stages consisting of meronts and sporonts (Fig. 2b-1). Early pupal stage ( $8 \mathrm{dpi}$ ) showed meronts containing two nuclei, diplokaryotic sporonts and sporonts close to division/ separation of nuclei (Fig. 2b-2). Later in pupal stage (10 dpi), few spores and predominantly (dividing) sporonts were observed (Fig. 2b-3. In late pupal stage (13 dpi), mainly spores could be found, but also sporoblasts (Fig. 2b-4). Adult stages (15-17 dpi) contained (Fig. 2b-5) mostly binucleate sporoblasts and single spores (Fig. 2b-6).

Eclosion of inoculated SWD larvae. L2 larvae exposed to $5 \times 10^{4} \mathrm{~T}$. suzukii spores showed $71.2 \%$ reduced eclosion at $19 \mathrm{dpi}($ mean $\pm \mathrm{SE}=19.84 \pm 8.41 \%)$ compared to the control $(69.1 \pm 4.14)$ (Fig. 3a). Eclosion at lower spore dosages did not statistically differ from the control group, though an intermediate mortality between those of the control and $5 \times 10^{3}$ spores was observed for the treatment with $5 \times 10^{2}$ spores GLM analysis for quasi- 
a

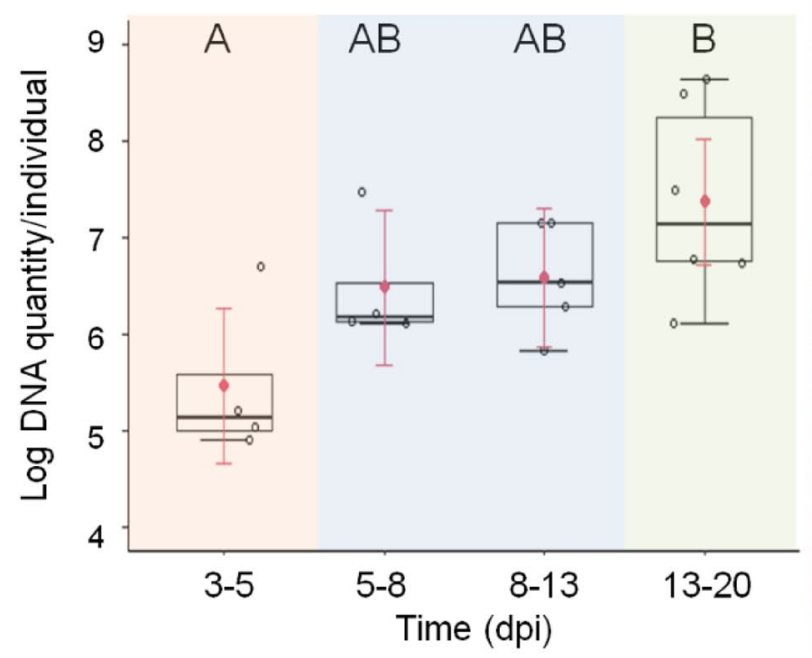

b

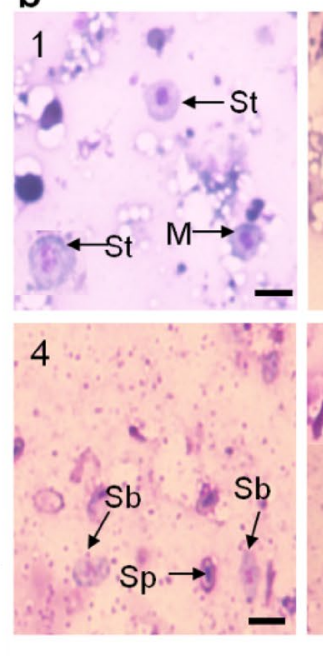

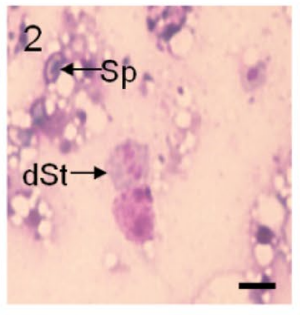

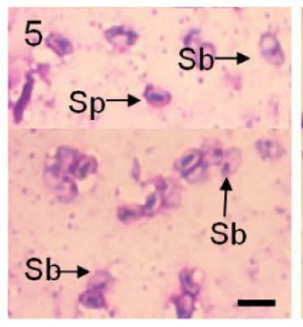

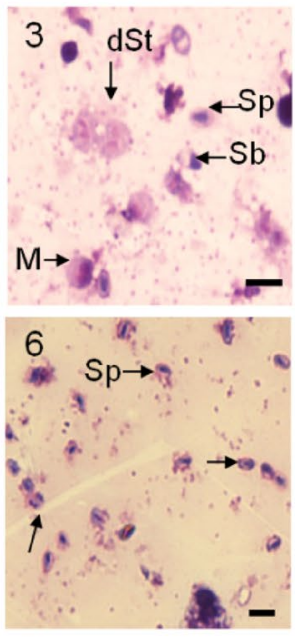

Figure 2. (a) Copy numbers of small subunit ribosomal DNA (SSU rDNA) (mean = red dot) of T. suzukii in single D. suzukii (SWD) larvae (orange), pupae (blue) and adults (green). The course of infection for second instar larvae (L2) inoculated with $4 \times 10^{4}$ spores. Replicates/numbers (R/N): larvae 3-5: $\mathrm{R} / \mathrm{N}=2 / 4$, pupae 5-8: $\mathrm{R} / \mathrm{N}=2 / 4$, pupae $8-13: \mathrm{R} / \mathrm{N}=3 / 5$, adult $13-20: \mathrm{R} / \mathrm{N}=3 / 6$. GLM analysis for gamma distribution with $\log$ link: $\chi^{2}=14.014, D F=3, P=0.003$, Tukey test: $3-5$ and 13-20: $P<0.001,5-8$ and 13-20: $P=0.252,8-13$ and 13-20: $P=0.305$. Different letters indicate statistical difference. Boxplot indicates the median logarithmic DNA quantity per individual (horizontal bar), mean (red dot), error bars (black hinge), asymptotic upper and lower confidence limits (red hinge) outliers (white dot). (b) Bright-field light microscopy of Giemsa-stained smears. Developmental stages of T. suzukii isolated from SWD larvae, pupae and adults at different time points post inoculation. SWD were inoculated in L2 larval stage with $4 \times 10^{4} \mathrm{~T}$. suzukii spores. (1) Larval stage (6 dpi). (2) Early pupal stage (8 dpi). (3) Pupal stages (10 dpi). (4) Late pupal stage (13 dpi). (5) Early adult stage (15 dpi). (6) Adult stage (17 dpi). Abbreviations: (M) meront containing two nuclei, (St) sporont, (Sp) spore, (dSt) dividing sporont, $(\mathrm{Sb})$ sporoblast. Scale bar $=5 \mu \mathrm{m}$.

binomial model with over-dispersion: $F=8.91, D F=3, P(\mathrm{~F})<0.001$, Tukey test: Control $-5 \times 10^{2}: P=0.889$, Control $-5 \times 10^{3}: P=0.226$, Control $-5 \times 10^{4}: P<0.0001$ ), (Fig. 3a).

For host range testing of cosmopolitan and exotic drosophilids, L2 larvae of D. melanogaster (DM, cosmopolitan) and D. willistoni (DW, exotic) were exposed to $5 \times 10^{4}$ of $T$. suzukii spores/well. This spore concentration did not significantly reduce eclosion in DM and DW compared to their corresponding controls (GLM analysis for quasi-binomial model with over-dispersion: $\mathrm{DM}, F=1.361, D F=1, P(\mathrm{~F})=0.261$; $\mathrm{DW}, F=1.987, D F=1$, $P(\mathrm{~F})=0.232$ ), (Fig. 3b).

Survival rates of inoculated SWD larvae and adults. Kaplan-Meier survival analyses were performed with individuals from the same experiment, which survived to the adult stage (Fig. 4a). Eclosion occurred from day 13 to day 19 as a result to some age differences among inoculated L2 larvae. Survival analysis was performed using log rank test with Bonferroni adjustment and revealed significant differences in the life span of SWD adults for every treatment compared to the control (Survival formula: $\chi^{2}=534, D F=3, P<0.001$ ) (Fig. $4 \mathrm{a}$ ). It was striking that the survival curves of the control group and the group treated with $5 \times 10^{2}$ spores were similar until day 35 , then mortality increased in the treated group, indicating a delayed effect on the survival of adults when inoculated with very low spore concentration as larvae.

To study the effect of late infection initiation, newly eclosed SWD adults were treated with $3 \times 10^{5}$ spores in $100 \mu$ l. In Kaplan-Meier analyses (Fig. 4b), survival rates of control males was significantly higher than infected males $(P=0.027)$, infected females $(P=0.001)$ and control females $(P=0.004)$, whereas control females had similar survival curves as infection treatments for both males and females $\left(\chi^{2}=13.1, D F=3, P=0.004\right)$.

Fecundity and fertility of SWD treated with $T$. suzukii. When SWD larvae were infected, the mean number $( \pm$ SEM) of eggs oviposited per female was $135.97 \pm 8.81$ for the untreated control pairs (Table 1). Pairs with infected parental females deposited $51 \%$ less eggs compared to control. For pairs with infected parental males the reduction was $48 \%$ and approximately $71 \%$ reduction in oviposition when both parents were infected (Table 1). For pairs with at least one infected parent, oviposition differed significantly from the untreated control, indicating a strong effect of microsporidium infection on the fecundity of $\operatorname{SWD}\left(\chi^{2}=48.84, D F=3, P<0.05\right.$, Dunn's test: F-C: $P=0.006$, M-C: $P=0.019$, MF-C: $P<0.001$ ). The mean lifetime (Table 1$)$ of pairs did not differ from each other $\left(\chi^{2}=2.683, D F=3, P=0.44\right)$, indicating parental longevity was not a factor in the differences in fecundity and fertility among infected and control SWD.

Comparing the number of eggs and resulting offspring within each treatment, no reduced hatching rates were observed (Table 1). Indeed, the number of viable offspring was very similar to number of eggs, thus the primary 

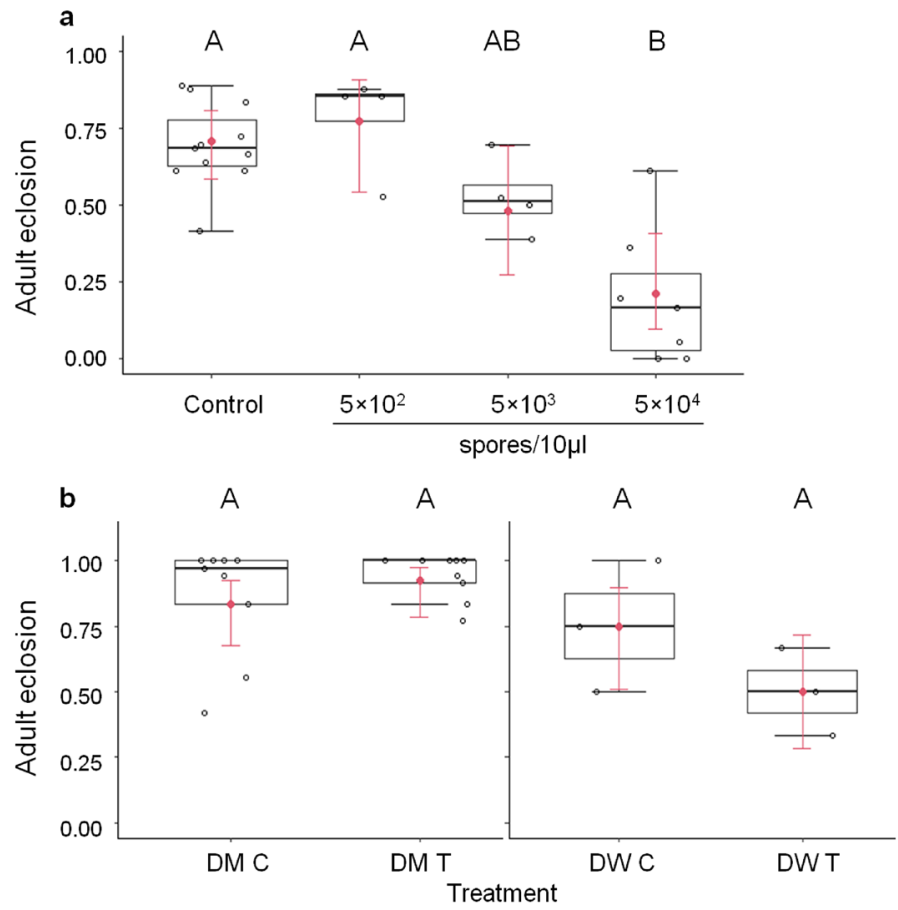

Figure 3. (a) D. suzukii adult eclosion (proportion, mean = red dot) at $19 \mathrm{dpi}$ after exposure to different $T$. suzukii spore dosages $\left(5 \times 10^{2}, 5 \times 10^{3}, 5 \times 10^{4}\right.$ spores/10 $\left.\mu \mathrm{l}\right)$ in second instar (L2) larval stage and controls. Replicate/number $(\mathrm{R} / \mathrm{N})$ : $\mathrm{C}=11 / 408,5 \times 10^{2}=4 / 180,5 \times 10^{3}=4 / 204,5 \times 10^{4}=7 / 264, F=8.91, D F=3, P(\mathrm{~F})<0.001$. (b) Eclosion (proportion) of D. melanogaster (DM T) and D. willistoni (DW T) exposed to $5 \times 10^{4}$ T. suzukii spores and corresponding controls (DM C, DW C) in L2 larval stage (replicates/total individuals (R/N), DM $\mathrm{C}: \mathrm{R} / \mathrm{N}=9 / 287, \mathrm{DM} \mathrm{T}: \mathrm{R} / \mathrm{N}=9 / 276, F=1.361, D F=1, P(\mathrm{~F})=0.261 ; \mathrm{DW}$ control: $\mathrm{R} / \mathrm{N}=3 / 48$, DW T: $\mathrm{R} / \mathrm{N}=3 / 48$, $F=1.987, D F=1, P(F)=0.232$ ). Different letters indicate statistical difference (see Fig. 2 for boxplot indication).

effect was on fecundity and not on fertility $\left(\chi^{2}=41.336, D F=3, P<0.05\right.$, Dunn's test (Bonferroni adjustment): F-C: $P=0.037$, M-C: $P=0.073$, MF-C: $P<0.001)$.

For control females, $45 \%$ of total eggs oviposited were laid in the first 10 days after eclosion (Table 1). Treatments of pairs including infected females differed significantly from the untreated control, but not pairs with only males infected $(F=13.96, D F=3, P(F)<0.001$, Tukey HSD: F-C: $P<0.05$, M-C: $P>0.05$, MF-C: $P<0.001)$.

The sex ratio of the offspring was not affected in female treatments $\left(\chi^{2}=0.45, D F=1, P=0.50\right)$ and trials with biparental $\left(\chi^{2}=0.057, D F=1, P=0.81\right)$ infection compared to controls $\left(\chi^{2}=0.37, D F=1, P=0.54\right)$; male to female ratio was close to 1:1 (Table 1). Male-only infection treatments revealed a sex-biased effect with significant more female offspring $\left(\chi^{2}=9, D F=1, P=0.003\right)$ (Table 1$)$. Microscopic inspection of $227 \mathrm{~F} 1$ individuals from transmission experiments with both infected parents $(\mathrm{R} / \mathrm{N}=2 / 18)$ revealed eight adult flies with a microsporidian infection (3.52\%) 3 weeks after eggs laid.

When SWD adults were inoculated with T. suzukii, mean oviposition was approximately $30 \%$ less in microsporidian-treated groups (MF) compared to the control groups (C) (Table 2), but no significant difference between both treatments was noted. Also, the number of eclosed offspring recorded 18 days after oviposition did not differ among treatments (Table 2). In both treatments, fertility was about 15-20\% lower than fecundity but this difference was not significant (Table 2).

The sex ratio of offspring from T. suzukii inoculated parents was shifted to female offspring $\left(\chi^{2}=9.09, D F=1\right.$, $P=0.003$ ), control treatments showed a sex ratio close to $1: 1\left(\chi^{2}=0.46, D F=1, P=0.50\right)$ (Table 2). Inspection of $223 \mathrm{~F} 1$ adults derived from T. suzukii treatment did not show any infected offspring, indicating that the microsporidium is not vertically transmitted if infection occurs in adult stage. Microscopic inspection of 31 Microsporidia-treated pairs resulted in one male fly with an established T. suzukii-infection.

\section{Discussion}

A novel microsporidium T. suzukii was discovered in 2015 in laboratory-reared SWD ${ }^{39}$. In this study, the potential impact of T. suzukii infection on SWD larvae and adults has been investigated and significant concentrationdependent effects on the mortality as well as the eclosion, survival, lifetime fecundity and effects on offspring were analysed when L2 larvae were inoculated with the microsporidium.

We succeeded in measuring the infection progress in individual SWD inoculated in larval stage by qPCR, which allowed us following the replication cycle of T. suzukii upon emergence of flies. Within the first 13 days post inoculation, DNA copies of microsporidian SSU rDNA increased up to 1000-fold, indicating strong microsporidian proliferation and explaining low pupal survival and adult eclosion after exposure to a high number 

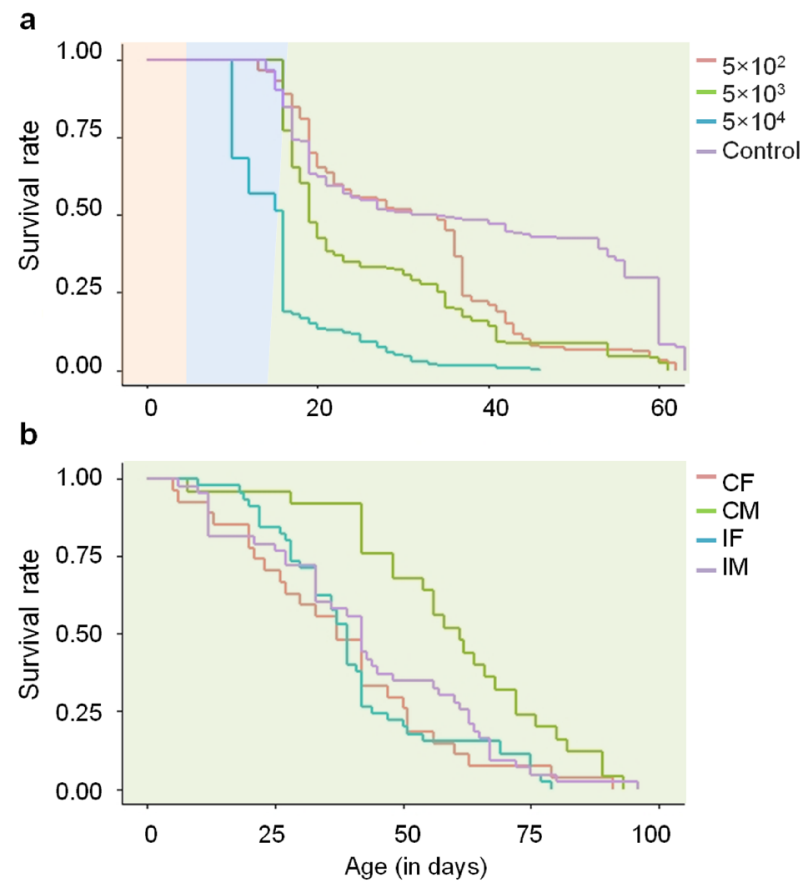

Figure 4. (a) Kaplan-Meier survival curves of D. suzukii (SWD), shown as complete lifespan from larval stage (day 0-6, orange field) to pupal stage (day 7-19, blue field) up to eclosion and death in adult stage (green field). Second instar larvae were either exposed to $5 \times 10^{2}$ spores (red line, replicates/number $\left.(\mathrm{R} / \mathrm{N})=4 / 180\right)$, $5 \times 10^{3}$ spores (green line, $\mathrm{R} / \mathrm{N}=4 / 204$ ), $5 \times 10^{4}$ spores (turquoise line, $\mathrm{R} / \mathrm{N}=7 / 264$ ) or sterile water as control (purple line, $\mathrm{R} / \mathrm{N}=11 / 408$ ). Log rank test: $\chi^{2}=534, D F=3, P<0.001$. (b) Kaplan-Meier survival curves of adult SWD inoculated with T. suzukii 3 days after emergence. Separated male and female SWD could either feed on sterile water as control $(\mathrm{CF}=$ control female, red, replicates/number $(\mathrm{R} / \mathrm{N})=4 / 27 ; \mathrm{CM}=$ control male, green, $\mathrm{R} / \mathrm{N}=4 / 25)$ or a spore suspension of $3 \times 10^{5} \mathrm{~T}$. suzukii spores $(\mathrm{IF}=$ inoculated female, turquoise, $\mathrm{R} / \mathrm{N}=4 / 45$; $\mathrm{IM}=$ inoculated male, purple, $\mathrm{R} / \mathrm{N}=4 / 43)$. Log rank test: $\chi^{2}=13.1 \mathrm{DF}=3, P=0.004$.

\begin{tabular}{|c|c|c|c|c|c|c|c|c|c|c|c|c|c|c|}
\hline ID & Treatment & $R / N$ & $\begin{array}{l}\begin{array}{l}\text { Life-time } \\
\text { (days) }\end{array} \\
\end{array}$ & SEM & $\begin{array}{l}\text { Eggs/pair/ } \\
\text { lifetime (\$) }\end{array}$ & SEM & $\begin{array}{l}\text { Eggs/ } \\
\text { pair/10 days } \\
(\%)(\$)\end{array}$ & SEM & $\begin{array}{l}\text { Offspring } \\
\text { (§) }\end{array}$ & SEM & $\begin{array}{l}\text { P (eggs/ } \\
\text { lifetime- } \\
\text { offspring) }\end{array}$ & \begin{tabular}{|l|}
$\begin{array}{l}\text { Sex ratio } \\
\text { offspring } \\
\text { / }\end{array}$ \\
\end{tabular} & $\begin{array}{l}\mathrm{N}(\text { sex } \\
\text { ratio) }\end{array}$ & $\begin{array}{l}\text { P (sex } \\
\text { ratio) }\end{array}$ \\
\hline C & $\mathrm{u} \varphi+\mathrm{uu}_{0}{ }^{-}$ & $3 / 40$ & 35.9 & 2.1 & $135.9(\mathrm{~A})$ & 8.8 & $\begin{array}{l}61.3(45.1) \\
(\mathrm{A})\end{array}$ & 5.4 & 127.7 (A) & 9.2 & $\begin{array}{r}>0.05 \\
T=1.09\end{array}$ & $0.49 / 0.51$ & 3351 & $\begin{array}{r}>0.05, \\
\chi^{2}=0.37\end{array}$ \\
\hline $\mathrm{F}$ & i $q \times \mathbf{u}^{\wedge}$ & $3 / 8$ & 32.8 & 3.5 & 65.4 (B) & 22.8 & $\begin{array}{l}30.4(46.5) \\
\text { (B) }\end{array}$ & 10.6 & 64.5 (B) & 22.9 & $\begin{array}{r}>0.05, \\
T=0.03\end{array}$ & $0.47 / 0.53$ & 179 & $\begin{array}{r}>0.05, \\
\chi^{2}=0.45\end{array}$ \\
\hline M & $\mathrm{u}+{ }^{\circ} \times \mathrm{i}_{0}{ }^{\top}$ & $3 / 8$ & 26.8 & 5.7 & 70.4 (B) & 19.9 & $\begin{array}{l}40.0(56.8) \\
(\mathrm{AB})\end{array}$ & 12.3 & $69.1(\mathrm{AB})$ & 19.9 & $\begin{array}{r}>0.05, \\
T=0.04\end{array}$ & $0.64 / 0.36$ & 121 & $\begin{array}{r}<0.05, \\
\chi^{2}=9.00\end{array}$ \\
\hline \multirow[t]{2}{*}{ MF } & 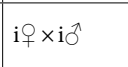 & $3 / 35$ & 29.6 & 1.5 & 39.8 (B) & 4.5 & $\begin{array}{l}19.4(48.7) \\
(\mathrm{B})\end{array}$ & 2.8 & $32.2(\mathrm{~B})$ & 4.8 & $\begin{array}{c}>0.05 \\
W=732.5\end{array}$ & $0.52 / 0.48$ & 439 & $\begin{array}{r}>0.05, \\
\chi^{2}=0.06\end{array}$ \\
\hline & $P$, test & & $\begin{array}{l}>0.05 \\
\chi^{2}=2.68\end{array}$ & & $\begin{array}{l}<0.001 \\
\chi^{2}=48.84\end{array}$ & & $\begin{array}{l}<0.001 \\
F=13.96\end{array}$ & & $\begin{array}{l}<0.05 \\
\chi^{2}=41.34\end{array}$ & & & & & \\
\hline
\end{tabular}

Table 1. Lifetime, fecundity (oviposition) and fertility (offspring) of SWD pairs inoculated with T. suzukii spores at larval stage. Sex ratio of resulting offspring was determined after eclosion of progeny. ID $=$ treatment $(\mathrm{C}=$ untreated control, $\mathrm{F}=$ female infected, $\mathrm{M}=$ male infected, $\mathrm{MF}=$ both infected $)$, treatment: $q=$ female, $\hat{\delta}=$ male, $\mathrm{u}=$ uninfected, $\mathrm{i}=$ infected, $\mathrm{R} / \mathrm{N}=$ number of replicates and total pairs, $(\S)=$ significance letter $(<0.05)$, SEM = standard error of mean, $\mathrm{N}$ (sex ratio) = total number of offspring analysed for sex. Lifetime, eggs/pair/lifetime, eggs/pair/10 days, offspring are shown as means.

of spores. Although we counted a maximum of $10^{6}$ spores when dissecting flies, quantification of DNA copies suggested even up to $10^{8}$ spores per fly in late infection stages. We found high larval mortality, whereas eclosion declined dramatically (up to 70\%) with increasing inoculated spore concentration. It has to be emphasized that the used amount of spores was experimentally motivated, but no data are available if such doses would be achieved in natural transmission scenarios when spores are released from dead individuals. SWD larvae surviving the T. suzukii infection would die during pupal or in early adult stages compared to controls. At high spore concentration, only $30 \%$ of flies survived the adult stage and these died within 40 days compared to 65 days in 


\begin{tabular}{|c|c|c|c|c|c|c|c|c|c|c|}
\hline ID & Treatment & $\mathrm{R} / \mathrm{N}$ & $\begin{array}{l}\text { Eggs/pair/lifetime in } \\
\text { days (\$) }\end{array}$ & SEM & Offspring (§) & SEM & $P$ (eggs-offspring) & $\begin{array}{l}\text { Sex ratio offspring } \\
+1 \widehat{\delta}^{\hat{n}}\end{array}$ & $N$ (sex ratio) & $\begin{array}{l}P(\text { sex } \\
\text { ratio })\end{array}$ \\
\hline $\mathrm{C}$ & $\mathbf{u} \varphi \times \mathbf{u}_{0} \hat{O}^{-}$ & $4 / 17$ & $152.9(\mathrm{~A})$ & 19.43 & $132.2(\mathrm{~A})$ & 19.3 & $\begin{array}{r}>0.05 \\
T=0.75\end{array}$ & $0.51 / 0.49$ & 1139 & $\begin{array}{r}>0.05, \\
\chi^{2}=0.46\end{array}$ \\
\hline \multirow[t]{2}{*}{ MF } & 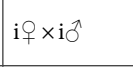 & $4 / 31$ & $107.6(\mathrm{~A})$ & 12.43 & $88.0(\mathrm{~A})$ & 10.6 & $\begin{array}{c}>0.05 \\
W=573.5\end{array}$ & $0.54 / 0.46$ & 1405 & $\begin{array}{r}<0.05, \\
\chi^{2}=9.08\end{array}$ \\
\hline & $P$, test & & $\begin{array}{l}0.059 \\
T=-1.96\end{array}$ & & $\begin{array}{l}0.055 \\
T=-2.01\end{array}$ & & & & & \\
\hline
\end{tabular}

Table 2. Lifetime, fecundity (oviposition) and fertility (offspring) of SWD pairs inoculated with T. suzukii spores at adult stage. Sex ratio of resulting offspring was determined after eclosion of progeny. ID = treatment abbreviation $(\mathrm{C}=$ untreated control, $\mathrm{MF}=$ both infected $)$, treatment: $+=$ female, $\hat{\delta}=$ male, $\mathrm{u}=$ uninfected, $\mathrm{i}=$ infected, $\mathrm{R} / \mathrm{N}=$ number of replicates and total pairs, $(\$)=$ significance letter $(<0.05)$, SEM $=$ standard error of mean, $\mathrm{N}$ (sex ratio) = total number of offspring analysed for sex. Lifetime, eggs/pair/lifetime, eggs/pair/10 days, offspring are shown as mean.

control. At low spore concentration, differences between the inoculated and control groups were not seen until day 35, when mortality increased. There was no evidence that T. suzukii has such strong effect on eclosion of other drosophilids when L2 larvae were inoculated. Compared to controls, eclosion of $D$. willistoni was reduced about $33 \%$ when larvae were inoculated with $5 \times 10^{4}$ spores but $D$. melanogaster was not affected at all. Virulence of $T$. suzukii to these two drosophilids was low, but transmission efficiency as another important infection parameter ${ }^{40}$ was not determined for both in this study.

When adults of SWD were inoculated, male control flies had a significantly longer lifespan compared to other treatments. This effect could be due to the experimental design where one female and one male were kept together. As shown for other drosophilids, mating significantly reduces survival of females and the absence of rivals results in increased male lifespan ${ }^{41-43}$. The high larval and lower adult susceptibility of SWD are in line with findings from other microsporidia of drosophilids, such as Tubulinosema ratisbonensis and Tubulinosema kingi $i^{23,44-49}$.

Fecundity and fertility were significantly reduced after larval inoculation resulting in about $70 \%$ reduction of egg deposits in biparental infections, but not after adult treatment. Females appeared to be the mainly weakened, as SWD pairs with only females infected had the second highest reduction of egg deposits. One important fitness parameter is the oviposition rate within the first 10 days after adult emergence, when drosophilids are laying the majority of eggs compared to the rest of the lifetime ${ }^{50}$. SWD pairs inoculated with T. suzukii laid about $46-57 \%$ of the lifetime-eggs within the first 10 days, whereas untreated controls deposited $45 \%$. Selection studies with continuous exposure of D. melanogaster to microsporidia showed increased longevity and higher early-life fecundity rates in selection lines when infected with the pathogen. In contrast, longevity of controls challenged with microsporidia was significantly shorter compared to the selection line, whereas the lifetime fecundity was higher in absence of the pathogen ${ }^{51}$. In our study, the sublethal concentrations for this experiment (single exposure of $10 \mu \mathrm{l}$ with $1.5 \times 10^{4}$ spores/ $\mu$ l) did not reduce adult fly survival. Hence, we observed no significantly higher oviposition than in controls within $10 \mathrm{dpi}$, comparable with results of earlier studies for T. kingi-infected D. melanogaster ${ }^{4}$. We found no change in fertility, all treatments showed only $10 \%$ reduced hatching of offspring assuming T. suzukii has no effect on fertility. No tissue-pathological evidence for T. suzukii infections in male gonads or sperms was found in a recent study, but sample size was low ${ }^{39}$. However, the combination of parental pairs with male-only infection $\left(\mathrm{M}: \mathrm{u}_{+} \times \mathrm{i}^{\top}{ }^{\top}\right)$ resulted in reduced oviposition as well. We found a statistically significant shift to higher proportions of female offspring (0.64) compared to controls (0.49) in this experimental setup, suggesting a parthenogenetic effect due to microsporidian infection. Since this effect was found from only three infected pairs more replicates would be useful to further support this finding. To our knowledge, pathogen-induced parthenogenesis was never described for SWD, although Wolbachia bacteria are known to induce female-biased progeny for approximately 40 drosophilid species ${ }^{52-56}$. The number of progeny in male-only treatments was comparably low to female-only treatment. Previous studies reported unmated females producing unfertilized eggs have a reduced total number of progeny for some drosophilids species ${ }^{57,58}$.

The here discussed high larval and low adult infection effects were similar to previous results of Vijendravarma et al. on stage-specific susceptibility of $D$. melanogaster treated with $T$. kingi ${ }^{47}$. For invertebrate hosts, several resistance mechanisms after microsporidian exposure were reported, showing induced cellular immune response like encapsulation, melanisation, hemocyte production, and phagocytosis ${ }^{65,66}$. In Diptera (i.e. drosophilids and mosquitoes), microsporidia infection induced up-regulation of lysozym genes and AMP production ${ }^{65}$. In selection experiments with D. melanogaster challenged with the microsporidium T. kingi or the fungus Beauveria bassiana, similar effects were observed: Flies selected on the pathogens had increased fitness and higher intra-specific competitiveness under pathogen pressure compared to non-selected control lines in presence of pathogens ${ }^{51,67}$. Due to the relatedness of T. kingi and T. suzukii ${ }^{39}$, similar outcomes may hold for SWD. Hence, combined effects of potential resistance to T. suzukii as well as over-represented fitness costs with low adult susceptibility implicate that this microsporidium might be a difficult tool for SWD pest management. Above that, commercial field application of microsporidia for insect biocontrol is still a matter of debate. In the 1970s, Antonospora (Paranosema) locustae (formerly: Nosema locustae) was used for locust and grasshopper management ${ }^{25}$ but low and slow mortality, inefficiency for some target species, and insignificant efficacy resulted in an economic failure of this agent. 
Furthermore, we conclude T. suzukii is not transmitted transovarially as offspring infection was rarely observed, but is horizontally transmitted via cadavers or frass. Transovum transmission is also possible. In former studies of the authors, T. suzukii spores were found inside maturing SWD ovaries of early adult stage females, possibly influencing egg development ${ }^{39}$. Spores were not found inside mature ovaries but infecting adipose tissue surrounding the ovaries, which can be transferred via egg deposition ${ }^{39}$. Other studies revealed transmission rates of $1-11 \%$ in Drosophila showing a similar transmission pattern for Tubulinosema microsporidia infecting closely related drosophilids ${ }^{23,45}$. In experiments where adults were inoculated, no oviposition reduction or vertical transmission was observed, suggesting a delayed infection process ${ }^{59,60}$.

In our experiments, T. suzukii had a very strong impact on longevity and fecundity of SWD in the lab when early larval stages were infected. Based on the negative effects, SWD infection could have a population reducing effect but adult flies are no suitable targets of T. suzukii. As this microsporidium was only found in a laboratory population so far, further field populations of SWD should be screened for natural occurrence of T. suzukii and other potential pathogens for biological control of this fruit damaging fly.

\section{Material and methods}

Insect host rearing. Microsporidia-free D. suzukii (SWD) flies were maintained in $30 \times 30 \times 30 \mathrm{~cm}$ cages (BUGDORM, MEGAVIEW SCIENCE CO., Taiwan) with water, a diet for adult flies ( $1 \mathrm{~g}$ brewer's yeast and $1 \mathrm{~g}$ sucrose) and an artificial oviposition medium as described elsewhere $\left({ }^{39}\right.$, modified from Chabert, et al. $\left.{ }^{30}\right)$. The oviposition medium was replaced weekly. If larvae with synchronous development were needed, the medium was replaced every $4 \mathrm{~h}$. Insect rearing and subsequent biotests were performed under the following conditions: $22 \pm 1{ }^{\circ} \mathrm{C}, 50 \%$ relative humidity (r.H.), 16:8 h light:dark photoperiod. Microsporidia-free D. melanogaster (DM) and $D$. willistoni (DW) were maintained under same conditions as described for SWD.

Preparation of T. suzukii spores for SWD inoculation. T. suzukii was first isolated from a D. suzukii rearing originating from flies collected in Oregon, USA ${ }^{38}$. Adult D. suzukii were homogenized with a micro pestle, dissolved in distilled sterile water and filtered through four layers of gauze, then additionally filtered through a cotton filter disc with 12-15 $\mu \mathrm{m}$ particle retention (Grade 1288, Ø $90 \mathrm{~mm}$, SARTORIUS AG, Göttingen, Germany). Spores were precipitated by centrifugation at 10,000×g (Centrifuge $5424 \mathrm{R}$, EPPENDORF, Hamburg, Germany) and resuspended in $500 \mu$ sterile tap water. Spore concentration and purity was determined with a Thoma hemocytometer under phase contrast microscopy (DMRB, LEICA, Wetzlar, Germany), followed by dilution in sterile tap water to final concentrations as required for subsequent biotests.

Preparation of standards in real-time quantitative PCR (qPCR). T. suzukii spores were extracted from adult SWD carrying an infection with spores (about 3 weeks after initial inoculation of L2 larvae with $10 \mu \mathrm{l}$ spore suspension containing $1.5 \times 10^{4} \mathrm{~T}$. suzukii spores). To produce standard curves, ten flies were homogenized with a micro pestle. About $1 \times 10^{7}$ spores were purified with one filtration step through four layers of gauze mesh and a final purification with Percoll. For this purpose, $400 \mu \mathrm{l}$ spore suspension was overlaid on $1.6 \mathrm{ml} 75 \%$ Percoll (MERCK, Darmstadt, Germany) dissolved in $1 \times$ PBS in a 2-ml reaction tube and spun down for 20 min at $12,900 \times g$ and $15^{\circ} \mathrm{C}$ in an EPPENDORF centrifuge (5424R, EPPENDORF, Hamburg, Germany). The spores formed a band close to the bottom of the reaction tube. The spore band was washed twice in $1 \times \mathrm{PBS}$ at $15,000 \times g$ for $5 \mathrm{~min}$, and the resulting pellet was resuspended in distilled water. Afterwards, spores were inspected for purity under phase contrast microscopy (DMRB, LEICA, Wetzlar, Germany). For preparation of a qPCR standard, serial dilutions of purified spores were prepared with $10^{1}-10^{6}$ spores in $100 \mu$ distilled water.

SWD inoculation and DNA extraction for qPCR. $\quad$ L2 larvae of SWD were exposed to $10 \mu 1$ spore suspension with $4 \times 10^{4}$ spores in a microtiter plate overlaid on $440 \mu \mathrm{l}$ pureed apple. Every $2-3$ days three larvae and later pupae or adults were removed and euthanized with ethyl-acetate and surface sterilized with $0.05 \%$ sodium hypochlorite. One individual was examined visually for infection by light microscopy with $400 \times$ magnification (DMRB, LEICA, Wetzlar, Germany) and a smear was stained with modified Giemsa-stain according to Eberle et al. ${ }^{61}$. Two larvae per replicate were used for genomic DNA extraction as described above and then qPCR. This was repeated for adult SWD which were starved for $3 \mathrm{~h}$ followed by bulk feeding for $18 \mathrm{~h}$ with a spore suspension containing in total $5 \times 10^{5} \mathrm{~T}$. suzukii spores mixed with blue food colour (modified droplet feeding method from Hughes and $\mathrm{Wood}^{62}$ ). To each group of ten flies, $10 \mu \mathrm{l}$ spore suspension were provided. The time frame for euthanizing four flies per replicate (day 3, 5, 10, 18, 28, 38) was longer than for larval inoculation, as infection was sometimes delayed in adults. Two flies were prepared for microscopy and Giemsa-staining and two other were used for genomic DNA extraction after surface sterilization with sodium hypochlorite.

Sample and standard spore preparations (see "Preparation of standards in real-time quantitative PCR (qPCR)") were centrifuged at $15,000 \times g$ for $10 \mathrm{~min}$. The spore pellet was dissolved in $200 \mu \mathrm{l}$ CTAB lysis buffer (APPLICHEM, Darmstadt, Germany). Following addition of $200 \mathrm{mg}$ of glass beads (0.25-0.5 mm diameter, ROTH, Karlsruhe, Germany) spores were broken by bead beating in a tissue disrupter at $24 \mathrm{MHz}$ for $1 \mathrm{~min}$ (MP FASTPREP-24 Tissue and Cell Homogenizer, MP BIOMEDICALS, Eschwege, Germany). Lysis was performed by adding $2 \mu \mathrm{l}$ proteinase $\mathrm{K}(200 \mathrm{ng} / \mu \mathrm{l}$, BIORON GMBH, Römerberg, Germany) and incubating samples at $56{ }^{\circ} \mathrm{C}$ on a thermo shaker at $250 \mathrm{rpm}$ for $18 \mathrm{~h}$. DNA was extracted with a two-step phenol-chloroform extraction with 25:24:1 phenol:chloroform:isoamyl alcohol. DNA preparations were washed twice in chloroform and finally subjected to ethanol precipitation (96\% ethanol). DNA pellets were dissolved in $30 \mu$ l distilled water. Quantitative PCR reactions to amplify small ribosomal subunit DNA (SSU rDNA) were carried out with $2 \mu$ of DNA dissolved in distilled water and Maxima SYBR Green qPCR Master Mix (THERMO FISHER SCIENTIFIC GMBH, Darmstadt, Germany) using 12.5 $\mu$ l Master Mix, $1 \mu 10$ mM forward primer Tn37F (5'-CGAAGATTT 
AGCCATGCATGCT-3') and $1 \mu \mathrm{l} 10 \mathrm{mM}$ reverse primer Tn562R (5' -CCGCTTCGAATATAAGCATTGA-3') ${ }^{39}$ and $8.5 \mu \mathrm{l}$ Nuclease-free water per reaction with following reaction conditions: $94^{\circ} \mathrm{C}$ for 3 min initial denaturation, followed by 35 cycles of $94^{\circ} \mathrm{C}$ for $45 \mathrm{~s}, 50^{\circ} \mathrm{C}$ for $30 \mathrm{~s}, 72^{\circ} \mathrm{C}$ for $90 \mathrm{~s}$ and stepwise temperature increase from $50{ }^{\circ} \mathrm{C}$ to $94{ }^{\circ} \mathrm{C}$ in $0.5^{\circ} \mathrm{C}$ every $5 \mathrm{~s}$ (CFX96 TOUCH Real Time PCR Systems, BIO-RAD LABORATORIES $\mathrm{GMBH}$, Feldkirchen, Germany).

Larval inoculation for lethal concentration $\left(\mathrm{LC}_{50}\right)$, survival and fecundity tests. The cavities of a 96-well microtiter plate (GREINER BIO-ONE GMBH, Frickenhausen, Germany) were filled with $440 \mu$ l pureed apple overlaid with $10 \mu \mathrm{l}$ spore suspension (concentrations for each experiment are listed below). A single L2 larva (3 days-old) was placed in each well the microtiter plate. Four experimental set ups were conducted:

(a) $\mathrm{LC}_{50}$ determination: application of five different spore concentrations in logarithmic scale $\left(10^{1}\right.$ to $10^{5}$ spores per $\mu$ l) to L2 larvae in a single microtiter plate. For each concentration one microtiter plate was prepared to avoid contamination and spill-over of larvae to another treatment. Two to nine independent replicates were performed.

(b) Eclosion and survival experiments: application of either $5 \times 10^{2}, 5 \times 10^{3}$ or $5 \times 10^{4} / 10 \mu \mathrm{l} \mathrm{T}$. suzukii spores to each larva in one single microtiter plate. For each concentration one microtiter plate was prepared to avoid contamination. Four to eleven replicates were performed. For each independent replicate, a new spore suspension was prepared.

(c) Fecundity tests: application of $1.5 \times 10^{4} / 10 \mu \mathrm{T}$. suzukii spores to each larva in a single microtiter plate.

(d) Host range testing with $D$. melanogaster and D. willistoni: application of $5 \times 10^{4} / 10 \mu \mathrm{l} \mathrm{T}$. suzukii spores to each one L2 larva of one species in a single microtiter plate. Three to nine replicates were prepared.

Untreated controls were prepared in separate microtiter plates and contained the equivalent amount (10 $\mu$ l) of sterile tap water overlaid on $440 \mu \mathrm{l}$ pureed apple. All implemented microtiter plates of one replicate for infection treatment and controls were prepared identically using the same batch of L2 larvae and kept under same rearing conditions.

Experimental design for $\mathrm{LC}_{50}$, eclosion and survival tests. Microtiter plates containing inoculated larvae were transferred into cylindrical cages $(30 \mathrm{~cm}$ height, $25 \mathrm{~cm}$ diameter, closed with a nylon membrane) containing a water source, adult diet and oviposition medium (see 2.1) were changed twice a week. Mortality in $\mathrm{LC}_{50}$ tests until 18 days post inoculation (dpi), eclosion until 19 dpi and survival of adults until 63 dpi were recorded daily until all SWD died. $\mathrm{The}^{\mathrm{LC}} \mathrm{L}_{50}$ at 18 dpi was calculated using probit analysis. Mortality data were corrected for control mortality by Abbott formula ${ }^{63}$.

Experimental design for fecundity tests. For fecundity experiments, inoculated larvae pupated and eclosed and the adults were separated into male and female groups directly after eclosion for 3 days to avoid premature mating. One 3-day-old naive male and female adult were then placed together for mating and oviposition They were held in boxes $(6 \mathrm{~cm}$ height, $10 \mathrm{~cm}$ diameter $)$ containing a Petri dish $(3 \mathrm{~cm}$ diameter $)$ with oviposition medium prepared as described in 2.1. After $48 \mathrm{~h}$, the Petri dish with oviposition medium was replaced and eggs were counted from the oviposition medium. The oviposition medium was then placed in a separate box and eggs were maintained until hatch. This procedure was repeated every 2 days until the last SWD pair inoculated with T. suzukii died. Number of hatched offspring was determined 18 days after oviposition and sex ratios were recorded. Treated flies were post-hoc inspected for established infections and assigned to different

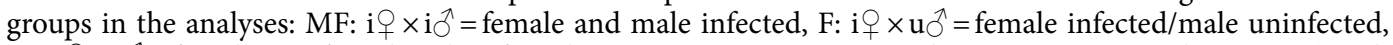
M: $\mathrm{u}^{\circ} \times \mathrm{i}^{\hat{\gamma}}=$ female uninfected/male infected. Transmission was examined in two separate trials (18 pairs) with both infected parents. Oviposition medium was changed every 2 days as described above and the oviposition rate was recorded. Eggs were transferred to fresh medium for development. Eggs were not surface sterilized to avoid manipulation of the respiratory filaments resulting in higher mortality. Experiments were carried out in an incubator (RUMED 3501, RUBARTH, Laatzen, Germany) under rearing conditions: $22 \pm 1{ }^{\circ} \mathrm{C}, 60 \% \mathrm{rH}, 16: 8 \mathrm{~h}$ light-dark photoperiod.

Inoculation of adults and design of survival and fecundity experiments. SWD adults at 3 days post-eclosion (survival experiment) or one day post-eclosion (fecundity experiment) were placed together in groups of four (survival experiment) or separated into groups of male-only and female-only groups (fecundity experiment) into a plastic box where they were starved for $3 \mathrm{~h}$ followed by bulk feeding with $100 \mu \mathrm{l}$ tap water droplets containing in total $3 \times 10^{5} \mathrm{~T}$. suzukii spores mixed with blue food colour over night (about $18 \mathrm{~h}$ ) (modified droplet feeding method from Hughes and Wood $^{62}$. Flies with a blue abdomen were selected for the experiments, whereby one male and one female were placed together in a cage (containing diet and water). For each independent replicate, a new spore suspension was prepared. Four replicates were prepared in total. In survival experiments, daily survival was recorded until all adults died. For fecundity experiments, egg deposition was recorded every 2 days and number of hatched offspring was determined 18 days post oviposition. Treated flies were post-hoc microscopically inspected to determine infection status.

Statistical analyses. Estimation of the median lethal concentration $\left(\mathrm{LC}_{50}\right)$ and slope of the concentrationmortality curve were calculated by probit analysis using TOXRAT software ${ }^{64}$. All other statistical analyses were conducted with R version 3.5.1 (2018-07-02). For test of normal distribution, Shapiro Wilk test was chosen with 
$\alpha=0.05$ level of significance. For qPCR DNA expression data and adult eclosion assays general linear models (GLM) were for gamma and quasi-binomial distributed data were used respectively. Numbers of independent replicates $(\mathrm{R})$ and sample size $(\mathrm{N})$ are given in the Results part. For all statistical tests a level of significance of $\alpha=0.05$ was applied if not indicated otherwise.

Author statements. The authors confirm that all methods described in the submitted manuscript "Infection effects of the new microsporidian species Tubulinosema suzukii on its host Drosophila suzukii" were carried out in accordance with relevant guidelines and regulations in the manuscript.

The authors confirm that all methods and experimental protocols used in the submitted manuscript "Infection effects of the new microsporidian species Tubulinosema suzukii on its host Drosophila suzukii" were approved by the Julius Kühn-Institut, Federal Research Centre for Cultivated Plants.

Received: 23 September 2020; Accepted: 22 April 2021

Published online: 12 May 2021

\section{References}

1. Capella-Gutiérrez, S., Marcet-Houben, M. \& Gabaldon, T. Phylogenomics supports microsporidia as the earliest diverging clade of sequenced fungi. BMC Biol. 10, 47. https://doi.org/10.1186/1741-7007-10-47 (2012).

2. Corsaro, D. et al. Filling gaps in the microsporidian tree: rDNA phylogeny of Chytridiopsis typographi (Microsporidia: Chytridiopsida). Parasitol. Res. 118, 169-180. https://doi.org/10.1007/s00436-018-6130-1 (2019).

3. Corsaro, D. et al. Molecular identification of Nucleophaga terricolae sp. nov. (Rozellomycota), and new insights on the origin of the Microsporidia. Parasitol. Res. 115, 3003-3011 (2016).

4. James, T. Y. et al. Reconstructing the early evolution of Fungi using a six-gene phylogeny. Nature 443, 818-822 (2006).

5. Sprague, V. \& Becnel, J. J. in The Microsporidia and Microsporidiosis (eds M. Wittner \& L. M. Weiss) 517-530 (ASM Press, 1999).

6. Dunn, A. M., Terry, R. S. \& Smith, J. E. Transovarial transmission in the microsporidia. Adv. Parasitol. 48, 57-100. https://doi.org/ 10.1016/S0065-308X(01)48005-5 (2001).

7. Goertz, D. \& Hoch, G. Vertical transmission and overwintering of Microsporidia in the gypsy moth, Lymantria dispar. J. Invertebr. Pathol. 99, 43-48. https://doi.org/10.1016/j.jip.2008.03.008 (2008).

8. Becnel, J. J. \& Andreadis, T. G. in Microsporidia: Pathogens of Opportunity (eds L. M. Weiss \& J. J. Becnel) 521-570 (Wiley, 2014).

9. Kellen, W. R. \& Lindegren, J. E. Modes of transmission of Nosema plodiae Kellen and Lindegren, a pathogen of Plodia interpunctella (Hübner). J. Stored Prod. Res. 7, 31-34. https://doi.org/10.1016/0022-474X(71)90035-X (1971).

10. Vávra, J. \& Larsson, R. J. in Microsporidia: Pathogens of Opportunity (eds L. M. Weiss \& J. J. Becnel) 1-70 (Wiley, 2014).

11. Mudasar, M., Mathivanan, V., Shah, G. N., Mir, G. M. \& Selvisabhanayakam, M. Nosemosis and its effect on performance of honey bees: A review. Int. J. Pharm. Bio. Sci. 4, 923-937 (2013).

12. Wolf, S. et al. So near and yet so far: Harmonic radar reveals reduced homing ability of Nosema infected honeybees. PLoS ONE 9 , e103989. https://doi.org/10.1371/journal.pone.0103989 (2014).

13. Naug, D. \& Gibbs, A. Behavioral changes mediated by hunger in honeybees infected with Nosema ceranae. Apidologie 40, 595-599 (2009).

14. Dussaubat, C. et al. Flight behavior and pheromone changes associated to Nosema ceranae infection of honey bee workers (Apis mellifera) in field conditions. J. Invertebr. Pathol. 113, 42-51 (2013).

15. Goblirsch, M., Huang, Z. Y. \& Spivak, M. Physiological and behavioral changes in honey bees (Apis mellifera) induced by Nosema ceranae infection. PLOS ONE 8, 6 (2013).

16. Lipsitch, M., Nowak, M. A., Ebert, D. \& May, R. M. The population dynamics of vertically and horizontally transmitted parasites. Proc. R. Soc. Lond. B 260, 321-327. https://doi.org/10.1098/rspb.1995.0099 (1995).

17. Goertz, D., Solter, L. F. \& Linde, A. Horizontal and vertical transmission of a Nosema sp. (Microsporidia) from Lymantria dispar (L.) (Lepidoptera: Lymantriidae). J. Invertebr. Pathol. 95, 9-16. https://doi.org/10.1016/j.jip.2006.11.003 (2007).

18. Kellen, W. R., Chapman, H. C., Clark, T. B. \& Lindegren, J. E. Host-parasite relationships of some Thelohania from mosquitoes (Nosematidae: Microsporidia). J. Invertebr. Pathol. 7, 161-166. https://doi.org/10.1016/0022-2011(65)90030-3 (1965).

19. Dunn, A. M. \& Smith, J. E. Microsporidian life cycles and diversity: the relationship between virulence and transmission. Microbes Infect. 3, 381-388. https://doi.org/10.1016/S1286-4579(01)01394-6 (2001).

20. Terry, R. S. et al. Widespread vertical transmission and associated host sex-ratio distortion within the eukaryotic phylum Microspora. Proc. R. Soc. Lond. B 271, 1783-1789. https://doi.org/10.1098/rspb.2004.2793 (2004).

21. Mercer, C. \& Wigley, P. A microsporidian pathogen of the poroporo stem borer, Sceliodes cordalis (Dbld)(Lepidoptera: Pyralidae): Effects on adult reproductive success. J. Invertebr. Pathol. 49, 108-115. https://doi.org/10.1016/0022-2011(87)90132-7 (1987).

22. Bauer, L. S. \& Nordin, G. L. Effect of Nosema fumiferanae (Microsporida) on fecundity, fertility, and progeny performance of Choristoneura fumiferana (Lepidoptera: Tortricidae). Environ. Entomol. 18, 261-265. https://doi.org/10.1093/ee/18.2.261 (1989).

23. Futerman, P. et al. Fitness effects and transmission routes of a microsporidian parasite infecting Drosophila and its parasitoids. Parasitology 132, 479-492. https://doi.org/10.1017/S0031182005009339 (2006).

24. Goertz, D., Golldack, J. \& Linde, A. Two different and sublethal isolates of Nosema lymantriae (Microsporidia) reduce the reproductive success of their host, Lymantria dispar. Biocontrol Sci. Technol. 18, 419-430. https://doi.org/10.1080/09583150801993212 (2008).

25. Lockwood, J. A., Bomar, C. R. \& Ewen, A. B. The history of biological control with Nosema locustae: Lessons for locust management. Int. J. Trop. Insect Sci. 19, 333-350. https://doi.org/10.1017/S1742758400018968 (1999).

26. Kiritani, K. \& Yamamura, K. in Invasive Species: Vectors and Management Strategies. (ed J. Carlton) 44-67 (Island Press, 2003).

27. Walsh, D. B. et al. Drosophila suzukii (Diptera: Drosophilidae): invasive pest of ripening soft fruit expanding its geographic range and damage potential. J. Integr. Pest Manage. 2, G1-G7. https://doi.org/10.1603/IPM10010 (2011).

28. Cini, A., Ioriatti, C. \& Anfora, G. A review of the invasion of Drosophila suzukii in Europe and a draft research agenda for integrated pest management. Bull. Insectol. 65, 149-160 (2012).

29. Tochen, S. et al. Temperature-related development and population parameters for Drosophila suzukii (Diptera: Drosophilidae) on cherry and blueberry. Environ. Entomol. 43, 501-510. https://doi.org/10.1603/en13200 (2014).

30. Chabert, S., Allemand, R., Poyet, M., Eslin, P. \& Gibert, P. Ability of European parasitoids (Hymenoptera) to control a new invasive Asiatic pest, Drosophila suzukii. Biol. Control 63, 40-47. https://doi.org/10.1016/j.biocontrol.2012.05.005 (2012).

31. Gabarra, R., Riudavets, J., Rodríguez, G., Pujade-Villar, J. \& Arnó, J. Prospects for the biological control of Drosophila suzukii. Biocontrol 60, 331-339. https://doi.org/10.1007/s10526-014-9646-z (2015).

32. Cuthbertson, A. G. S. \& Audsley, N. Further screening of entomopathogenic fungi and nematodes as control agents for Drosophila suzukii. Insects 7, 24. https://doi.org/10.3390/insects7020024 (2016). 
33. Woltz, J. M., Donahue, K. M., Bruck, D. J. \& Lee, J. C. Efficacy of commercially available predators, nematodes and fungal entomopathogens for augmentative control of Drosophila suzukii. J. Appl. Entomol. 139, 759-770. https://doi.org/10.1111/jen. 12200 (2015).

34. Haye, T. et al. Current SWD IPM tactics and their practical implementation in fruit crops across different regions around the world. J. Pest. Sci. 89, 643-651. https://doi.org/10.1007/s10340-016-0737-8 (2016).

35. Biganski, S., Jehle, J. A. \& Kleespies, R. G. Bacillus thuringiensis serovar israelensis has no effect on Drosophila suzukii Matsumura. J. Appl. Entomol. 142, 33-36. https://doi.org/10.1111/jen.12415 (2018).

36. Carrau, T., Hiebert, N., Vilcinskas, A. \& Lee, K.-Z. Identification and characterization of natural viruses associated with the invasive insect pest Drosophila suzukii. J. Invertebr. Pathol. 154, 74-78. https://doi.org/10.1016/j.jip.2018.04.001 (2018).

37. Medd, N. C. et al. The virome of Drosophila suzukii, an invasive pest of soft fruit. BioRxiv 4, 190322. https://doi.org/10.1093/ve/ vey009 (2017).

38. Kaur, R., Siozios, S., Miller, W. J. \& Rota-Stabelli, O. Insertion sequence polymorphism and genomic rearrangements uncover hidden Wolbachia diversity in Drosophila suzukii and D. subpulchrella. Sci. Rep. 7, 14815. https://doi.org/10.1038/s41598-017-13808-z (2017).

39. Biganski, S. et al. Molecular and morphological characterisation of a novel microsporidian species, Tubulinosema suzukii, infecting Drosophila suzukii (Diptera: Drosophilidae). J. Invertebr. Pathol. 107440 (2020).

40. Anderson, R. M. \& May, R. M. Coevolution of hosts and parasites. Parasitology 85, 411-426. https://doi.org/10.1017/S003118200 $0055360(1982)$

41. Aigaki, T. \& Ohba, S. Effect of mating status on Drosophila virilis lifespan. Exp. Gerontol. 19, 267-278. https://doi.org/10.1016/ 0531-5565(84)90022-6 (1984).

42. Partridge, L., Green, A. \& Fowler, K. Effects of egg-production and of exposure to males on female survival in Drosophila melanogaster. J. Insect Physiol. 33, 745-749. https://doi.org/10.1016/0022-1910(87)90060-6 (1987).

43. Bretman, A., Westmancoat, J. D., Gage, M. J. \& Chapman, T. Costs and benefits of lifetime exposure to mating rivals in male Drosophila melanogaster. Evolution 67, 2413-2422. https://doi.org/10.1111/evo.12125 (2013).

44. Armstrong, E. \& Bass, L. K. Nosema kingi: Effects on fecundity, fertility, and longevity of Drosophila melanogaster. J. Exp. Zool. 250, 82-86. https://doi.org/10.1002/jez.1402500111 (1989).

45. Armstrong, E. Transmission and infectivity studies on Nosema kingi in Drosophila willistoni and other Drosophilids. Z. Parasitenkd. 50, 161-165. https://doi.org/10.1007/BF00380520 (1976).

46. Armstrong, E., Bass, L., Staker, K. \& Harrell, L. A comparison of the biology of a Nosema in Drosophila melanogaster to Nosema kingi in Drosophila willistoni. J. Invertebr. Pathol. 48, 124-126. https://doi.org/10.1016/0022-2011(86)90151-5 (1986).

47. Vijendravarma, R. K., Godfray, H. C. J. \& Kraaijeveld, A. R. Infection of Drosophila melanogaster by Tubulinosema kingi: Stagespecific susceptibility and within-host proliferation. J. Invertebr. Pathol. 99, 239-241. https://doi.org/10.1016/j.jip.2008.02.014 (2008).

48. Niehus, S., Giammarinaro, P., Liégeois, S., Quintin, J. \& Ferrandon, D. Fly culture collapse disorder: Detection, prophylaxis and eradication of the microsporidian parasite Tubulinosema ratisbonensis infecting Drosophila melanogaster. Fly 6, 193-204. https:// doi.org/10.4161/fly.20896 (2012).

49. Franchet, A., Niehus, S., Caravello, G. \& Ferrandon, D. Phosphatidic acid as a limiting host metabolite for the proliferation of the microsporidium Tubulinosema ratisbonensis in Drosophila flies. Nat Microbiol 4, 645-655 (2019).

50. Robertson, F. W. \& Sang, J. H. The ecological determinants of population growth in a Drosophila culture. I. Fecundity of adult flies. Proc. R. Soc. Lond. B 132, 258-277. https://doi.org/10.1098/rspb.1944.0017 (1944).

51. Vijendravarma, R. K., Kraaijeveld, A. R. \& Godfray, H. C. J. Experimental evolution shows Drosophila melanogaster resistance to a microsporidian pathogen has fitness costs. Evolution 63, 104-114. https://doi.org/10.1111/j.1558-5646.2008.00516.x (2009).

52. Rousset, F., Bouchon, D., Pintureau, B., Juchault, P. \& Solignac, M. Wolbachia endosymbionts responsible for various alterations of sexuality in arthropods. Proc. R. Soc. Lond. B 250, 91-98. https://doi.org/10.1098/rspb.1992.0135 (1992).

53. Saeed, N., Battisti, A., Martinez-Sañudo, I. \& Mori, N. Combined effect of temperature and Wolbachia infection on the fitness of Drosophila suzukii. Bull. Insectol. 71, 161-169 (2018).

54. Hamm, C. A. et al. Wolbachia do not live by reproductive manipulation alone: infection polymorphism in Drosophila suzukii and D. subpulchrella. Mol. Ecol. 23, 4871-4885. https://doi.org/10.1111/mec.12901 (2014).

55. Mazzetto, F., Gonella, E. \& Alma, A. Wolbachia infection affects female fecundity in Drosophila suzukii. Bull. Insectol. 68, 153-157 (2015).

56. Hurst, G. D., Johnson, A. P., vd Schulenburg, J. H. G. \& Fuyama, Y. Male-killing Wolbachia in Drosophila: a temperature-sensitive trait with a threshold bacterial density. Genetics 156, 699-709 (2000).

57. Markow, T. A. Parents without partners: Drosophila as a model for understanding the mechanisms and evolution of parthenogenesis. G3 3, 757-762. https://doi.org/10.1534/g3.112.005421 (2013).

58. Wolfner, M. F. The gifts that keep on giving: physiological functions and evolutionary dynamics of male seminal proteins in Drosophila. Heredity 88, 85-93. https://doi.org/10.1038/sj.hdy.6800017 (2002).

59. Blaser, M. \& Schmid-Hempel, P. Determinants of virulence for the parasite Nosema whitei in its host Tribolium castaneum. J. Invertebr. Pathol. 89, 251-257. https://doi.org/10.1016/j.jip.2005.04.004 (2005).

60. Solter, L. F. in Microsporidia: Pathogens of Opportunity (eds L. M. Weiss \& J. J. Becnel) 165-194 (Wiley, 2014).

61. Eberle, K. E., Wennmann, J. T., Kleespies, R. G. \& Jehle, J. A. in Manual of Techniques in Invertebrate Pathology (ed L. A. Lacey) 15-74 (Academic Press, 2012).

62. Hughes, P. \& Wood, H. A synchronous peroral technique for the bioassay of insect viruses. J. Invertebr. Pathol. 37, 154-159. https:// doi.org/10.1016/0022-2011(81)90069-0 (1981).

63. Abbott, W. A method of computing the effectiveness of an insecticide. J. Econ. Entomol. 18, 265-267 (1925).

64. Software for the statistical analysis of biotests (ToxRat GmbH, Alsdorf, Germany, 2003).

65. Pan, G. et al. Invertebrate host responses to microsporidia infections. Dev. Comp. Immunol. 83, 104-113. https://doi.org/10.1016/j. dci.2018.02.004 (2018)

66. Roxström-Lindquist, K., Terenius, O. \& Faye, I. Parasite-specific immune response in adult Drosophila melanogaster: A genomic study. EMBO Rep. 5, 207-212. https://doi.org/10.1038/sj.embor.7400073 (2004).

67. Kraaijeveld, A. R. \& Godfray, H. C. J. Selection for resistance to a fungal pathogen in Drosophila melanogaster. Heredity 100, 400-406. https://doi.org/10.1038/sj.hdy.6801092 (2008).

\section{Acknowledgements}

Funding for this project was provided by the Federal Ministry of Food and Agriculture Germany (BMEL). The authors thank Jörg T. Wennmann for his advice in qPCR. Open Access funding enabled and organized by Projekt DEAL. 


\title{
Author contributions
}

S.B. designed and analysed the experiments and drafted the first version of the manuscript; S.B. and S.F. conducted experiments; R.G.K. and J.A.J. supervised the project; S.B., R.G.K. and J.A.J. wrote the final manuscript.

\section{Competing interests}

The authors declare no competing interests.

\section{Additional information \\ Correspondence and requests for materials should be addressed to R.G.K.}

Reprints and permissions information is available at www.nature.com/reprints.

Publisher's note Springer Nature remains neutral with regard to jurisdictional claims in published maps and institutional affiliations.

\begin{abstract}
(c) (1) Open Access This article is licensed under a Creative Commons Attribution 4.0 International License, which permits use, sharing, adaptation, distribution and reproduction in any medium or format, as long as you give appropriate credit to the original author(s) and the source, provide a link to the Creative Commons licence, and indicate if changes were made. The images or other third party material in this article are included in the article's Creative Commons licence, unless indicated otherwise in a credit line to the material. If material is not included in the article's Creative Commons licence and your intended use is not permitted by statutory regulation or exceeds the permitted use, you will need to obtain permission directly from the copyright holder. To view a copy of this licence, visit http://creativecommons.org/licenses/by/4.0/.
\end{abstract}

(C) The Author(s) 2021, corrected publication 2021 